\title{
COVID-19 related Attitudes and Risk Perceptions across Urban, Rural, and Suburban Areas in the United States
}

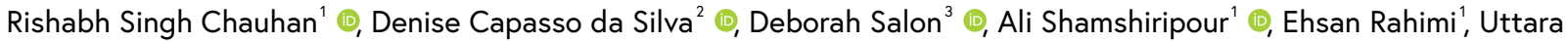

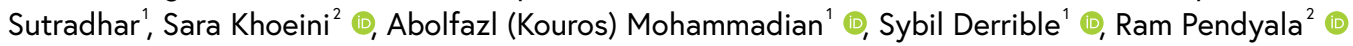 \\ ${ }^{1}$ Department of Civil, Materials, and Environmental Engineering, University of Illinois at Chicago, IL, USA, ${ }^{2}$ School of Sustainable Engineering and the Built \\ Environment, Arizona State University, Tempe, AZ, USA, ${ }^{3}$ School of Geographical Sciences and Urban Planning, Arizona State University, Tempe, AZ, USA \\ Keywords: covid-19 related attitudes, urban, suburban, rural, risk perception \\ https://doi.org/10.32866/001c.23714
}

Findings

This study identifies differences in COVID-19 related attitudes and risk perceptions among urban, rural, and suburban populations in the US using data from an online, nationwide survey collected during April-October 2020. In general, rural respondents were found to be less concerned by the pandemic and a lower proportion of rural respondents support staying at home and shutting down businesses. While only about half of rural respondents are concerned about getting severe reactions themselves from COVID-19 (compared to $60 \%$ for urban and suburban respondents), all place types respondents are concerned about friends or family members getting severe reactions ( $75 \%)$.

\section{Questions}

The COVID-19 pandemic has affected the entire world but its impacts have not been spread evenly (Zhang and Schwartz 2020). In the United States (US), urban areas initially reported a much higher proportion of COVID-19 related deaths, but they were later surpassed by rural areas (CDC 2020b). The Center for Disease Control and Prevention (CDC) has warned that populations in rural communities may be at a higher risk because they are generally older, have higher rates of chronic diseases, and have a higher proportion of the population with a disability (CDC 2020a). Several studies have also found differences in attitudes and behaviors toward COVID-19 varying between urban and rural areas (Boyle, Brassell, and Dayton 2020; Chen and Chen 2020; Haischer et al. 2020). The goal of this article is to investigate spatial variations in COVID-19 related attitudes and risk perception across the US among three groups of populations: urban, suburban, and rural.

\section{Methods}

The data were collected through an online longitudinal survey that asked a variety of COVID-19 related attitudinal and behavioral questions to more than 9,000 individuals from across the US (Capasso da Silva et al. 2021; Chauhan et al., 2021 (forthcoming); Conway et al. 2020; Salon et al. 2021). Specifically, we use the data collected during the first wave of the survey, which was conducted from April to October 2020. The survey data were weighted to match the distribution of the 2018 American Community Survey (ACS) 1-year estimates (Ruggles et al. 2020) using as control variables: age, education, gender, Hispanic status, household income, presence of children, and the number of household vehicles. More details on the data can be found in Chauhan et al. (2021 (forthcoming)). 
Table 1. Number and percentage of respondents in each place type category

\begin{tabular}{|l|c|c|}
\hline Place types & Number of survey responses (N) & Weighted percentage distribution \\
\hline Urban & 3,132 & $32.82 \%$ \\
\hline Suburban & 4,951 & $56.37 \%$ \\
\hline Rural & 605 & $10.81 \%$ \\
\hline Total & 8,688 & $100 \%$ \\
\hline
\end{tabular}

Using the respondents' current ZIP code, we categorized the sample into urban, suburban, and rural based on their housing densities, following the thresholds found by Kolko et. al. (2015). Categorizing ZIP codes based on housing density might be particularly useful for COVID-19 related analysis since it was found that increase in housing density increases the risk of COVID-19 (DiMaggio et al. 2020). Specifically, ZIP codes with a housing density above 2,213 households per square mile are classified as urban, those between 102 to 2,213 households per square mile as suburban, and those with fewer than 102 households per square mile as rural. Table 1 shows the distribution of respondents in each place type category.

The differences in attitudes and risk perception across the three place types were found through comparative analyses. Chi-squared tests were used to ensure that the differences are statistically significant. Furthermore, random forest (RF) models were estimated with attitudes and risk perception as dependent variables and place type variables and demographic variables (including the control variables) as independent variables. The feature importance (FI) of each variable was calculated to ensure that place type contributed to the differences in the attitudes and risk perceptions (not just the differences in demographic characteristics).

\section{Findings}

Significant differences were found in COVID-19 related attitudes and risk perception across respondents living in urban, suburban, and rural areas (Figures 1 and 2). More support for staying at home and shutting down the business was found in urban respondents, followed by suburban, and least in rural respondents. In particular, $79 \%$ of urban respondents support staying at home compared to $65 \%$ for rural respondents. $56 \%$ of urban respondents feel that shutting down the businesses to prevent the spread of the virus is worth the economic damage compared to $40 \%$ of rural respondents. About a third $(36 \%)$ of rural respondents feel that society is overreacting to coronavirus, compared to a quarter (26\%) of suburban respondents and a fifth (19\%) of urban respondents.

With 53\%, respondents from rural areas are slightly less concerned about getting severe reactions from COVID-19 compared to those from suburban $(58 \%)$ and urban $(61 \%)$ areas. Interestingly, across all three place types, the 


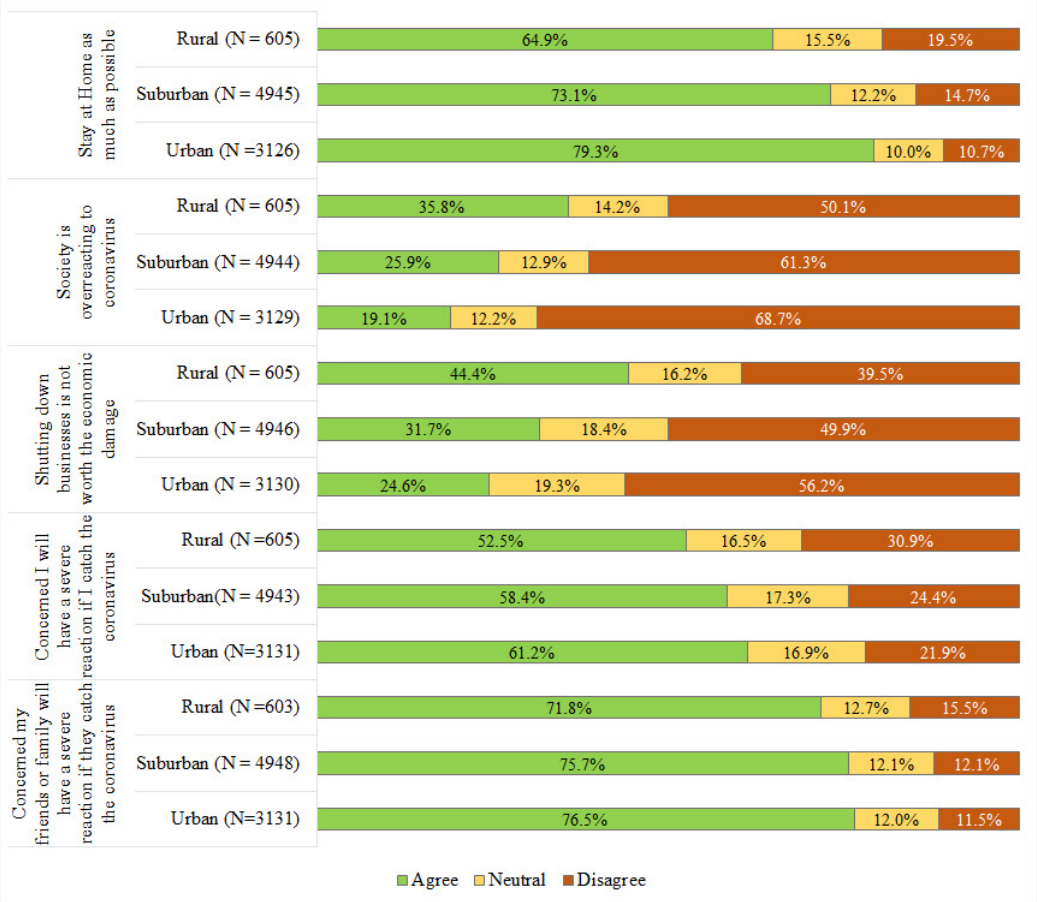

Figure 1. Differences in COVID-19 related attitudes across the urban, suburban, and rural respondents.

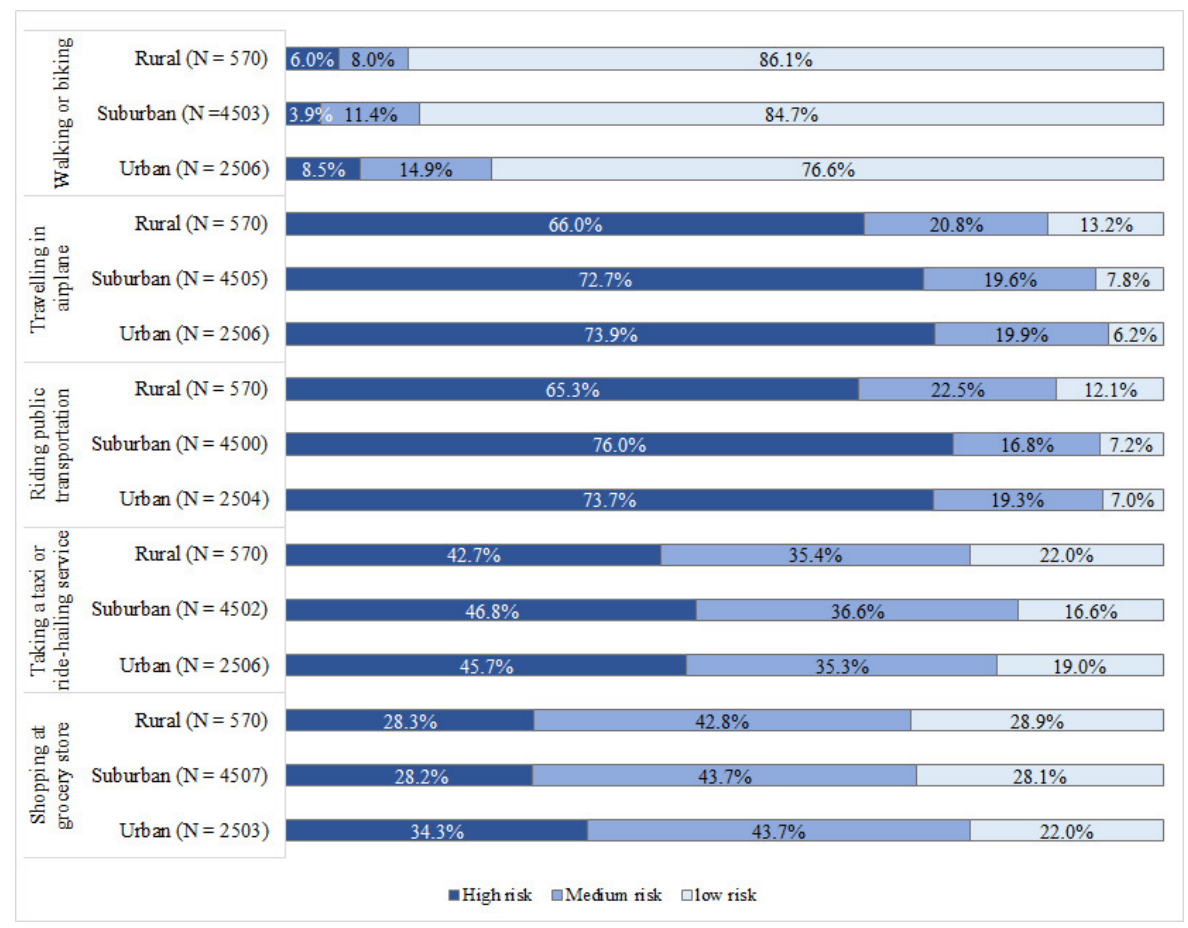

Figure 2. Difference in COVID-19 related risk perception across the urban, suburban, and rural respondents.

proportion of respondents having concern that friends or family members get severe reactions from COVID-19 was much higher than the same concern for themselves. 
Significant differences were found in COVID-19 related risk perceptions. Compared to suburban and rural respondents, urban respondents have the highest share of respondents who perceive high risk in shopping at grocery store (34\%) and walking or biking (9\%). Compared to urban and suburban respondents, rural respondents have the lowest percentage of respondents who perceive high risk in traveling by airplane (66\%) and public transit (65\%). Suburban respondents have the highest proportion of respondents who feel high risk in taking a taxi or ride-hailing service (47\%).

All of these differences were found to be statistically significant with a $95 \%$ confidence level using Chi-squared tests (please see Table A in supplementary materials for details). The RF models found that place type has a greater than zero FI for all variables. The FI of place type had a fairly high rank for the risk perception in taking taxi or ride hailing services. In contrast, it had a low rank for attitude regarding staying at home indicating that other demographic variables influence this attitude more than place type. (please see Table B in supplementary materials for details).

Overall, rural residents are found to be less concerned by the COVID-19 virus, while urban residents are more concerned. These findings corroborate with other studies (Boyle, Brassell, and Dayton 2020; Chen and Chen 2020; Haischer et al. 2020). Nonetheless, since COVID-19 cases and deaths have evolved since the completion of wave 1 of our survey (CDC 2020b), attitudes from urban, rural, and suburban populations may have evolved as well. The data collected from the subsequent survey waves (Chauhan et al., 2021 (forthcoming)) should help in exploring temporal variation in attitudes across locations. Furthermore, beyond attitude and risk perception, urban, suburban, and rural populations are often found to exhibit different behaviors (e.g., energy and reseource consumption (Derrible 2019)) that could be investigated to assess how differently the three populations were impacted by the COVID-19 pandemic.

\section{Acknowledgements}

This research was supported in part by the National Science Foundation (NSF) RAPID program under grants no. 2030156 and 2029962 and by the Center for Teaching Old Models New Tricks (TOMNET), a University Transportation Center sponsored by the U.S. Department of Transportation through grant no. 69A3551747116, as well as from the Knowledge Exchange for Resilience at Arizona State University. This COVID-19 Working Group effort was also supported by the NSF-funded Social Science Extreme Events Research (SSEER) network and the CONVERGE facility at the Natural Hazards Center at the University of Colorado Boulder (NSF Award \#1841338) and the NSF CAREER award under grant no. 155173. Any opinions, findings, and conclusions or recommendations expressed in this 
material are those of the authors and do not necessarily reflect the views of the funders. We would also like to acknowledge Matthew Wigginton Conway, $\mathrm{PhD}$ candidate at Arizona State University, for his meaningful inputs.

Submitted: March 09, 2021 AEST, Accepted: May 03, 2021 AEST

This is an open-access article distributed under the terms of the Creative Commons Attribution 4.0 International License (CCBY-SA-4.0). View this license's legal deed at https://creativecommons.org/ licenses/by-sa/4.0 and legal code at https://creativecommons.org/licenses/by-sa/4.0/legalcode for more information. 


\section{REFERENCES}

Boyle, John, Thomas Brassell, and James Dayton. 2020. "Rural Americans Feel Less Threatened by COVID-19 than Urban and Suburban Americans Do, but Still View Mitigation as Important." ICF. July 22, 2020. https://www.icf.com/insights/health/covid-19-survey-rural-vs-urban-threat.

Capasso da Silva, Denise, Sara Khoeini, Deborah Salon, Matthew Wigginton Conway, Rishabh Chauhan, Ram Pendyala, Ali Shamshiripour, et al. 2021. "How Do Attitudes Toward COVID-19 Affect Travel Behavior During the Pandemic?” Transport Findings.

CDC. 2020a. "COVID-19 and Your Health." Centers for Disease Control and Prevention. February 11, 2020. https://www.cdc.gov/coronavirus/2019-ncov/need-extra-precautions/other-atrisk-populations/rural-communities.html.

- - 2 2020b. "COVID Data Tracker." Centers for Disease Control and Prevention. March 28, 2020. https://covid.cdc.gov/covid-data-tracker.

Chauhan, Rishabh, Matthew Conway, Denise Capasso da Silva, Deborah Salon, Ali Shamshiripour, Ehsan Rahimi, Sara Khoeini, Abolfazl (Kouros) Mohammadian, Sybil Derrible, and Ram Pendyala. 2021 (forthcoming). "A Database of Travel-Related Behaviors and Attitudes Before, During, and After COVID-19 in the United States." Scientific Data.

Chen, Xuewei, and Hongliang Chen. 2020. "Differences in Preventive Behaviors of COVID-19 between Urban and Rural Residents: Lessons Learned from A Cross-Sectional Study in China.” International Journal of Environmental Research and Public Health 17 (12): 4437. https://doi.org/ $\underline{10.3390 / i j e r p h 17124437 .}$

Conway, Matthew Wigginton, Deborah Salon, Denise Capasso da Silva, and Laura Mirtich. 2020. "How Will the COVID-19 Pandemic Affect the Future of Urban Life? Early Evidence from Highly-Educated Respondents in the United States." Urban Science 4 (4): 50.

Derrible, Sybil. 2019. Urban Engineering for Sustainability. MIT Press.

DiMaggio, Charles, Michael Klein, Cherisse Berry, and Spiros Frangos. 2020. "Blacks/African Americans Are 5 Times More Likely to Develop COVID-19: Spatial Modeling of New York City ZIP Code-Level Testing Results.” MedRxiv 14: 2020.

Haischer, Michael H., Rachel Beilfuss, Meggie Rose Hart, Lauren Opielinski, David Wrucke, Gretchen Zirgaitis, Toni D. Uhrich, and Sandra K. Hunter. 2020. "Who Is Wearing a Mask? Gender-, Age-, and Location-Related Differences during the COVID-19 Pandemic." PLOS ONE 15 (10): e0240785. https://doi.org/10.1371/journal.pone.0240785.

Kolko, Jed. 2015. “How Suburban Are Big American Cities?” FiveThirtyEight (blog). May 21, 2015. https://fivethirtyeight.com/features/how-suburban-are-big-american-cities/.

Ruggles, Steven, Sarah Flood, Ronald Goeken, Erin Meyer, Jose Pacas, and Matthew Sobek. 2020. "IPUMS USA: Version 10.0 [Dataset].” 2020. https://doi.org/10.18128/D010.V10.0.

Salon, Deborah, Matthew Wigginton Conway, Denise Capasso da Silva, Rishabh Chauhan, Ali Shamshiripour, Ehsan Rahimi, Laura Mirtich, et al. 2021. "COVID Future Wave 1 Survey Data v0.9.0.” ASU Library Research Data Repository. https://dataverse.asu.edu/ dataset.xhtml?persistentId=doi:10.48349/ASU/HQYADA.

Zhang, Charlie H., and Gary G. Schwartz. 2020. "Spatial Disparities in Coronavirus Incidence and Mortality in the United States: An Ecological Analysis as of May 2020.” The Journal of Rural Health 36 (3): 433-45. https://doi.org/10.1111/jrh.12476. 


\section{SUPPLEMENTARY MATERIALS}

\section{Supplementary Material}

Download: https://findingspress.org/article/23714-covid-19-related-attitudes-and-risk-perceptionsacross-urban-rural-and-suburban-areas-in-the-united-states/attachment/60421.docx 